\title{
Long-Term Safety and Efficacy of Siluron2000 with Pars Plana Vitrectomy in the Treatment of Patients with Severe Vitreoretinopathy and Chronic Macular Holes
}

\author{
Parnian Arjmand ${ }^{1,2}$ \\ Tina Felfeli (iD) 1,3,4 \\ Efrem D Mandelcorn ${ }^{1,2,4}$ \\ Mark S Mandelcorn ${ }^{1,2,4}$ \\ 'Department of Ophthalmology and \\ Vision Sciences, University of Toronto, \\ Toronto, Ontario, Canada; ${ }^{2}$ Department \\ of Ophthalmology, Toronto Western \\ Hospital, University Health Network, \\ Toronto, Ontario, Canada; ${ }^{3}$ The Institute \\ of Health Policy, Management and \\ Evaluation (IHPME), Dalla Lana School of \\ Public Health, University of Toronto, \\ Toronto, Ontario, Canada; ${ }^{4}$ Faculty of \\ Medicine, University of Toronto, Toronto, \\ Ontario, Canada
}

Purpose: Silicone oil intraocular retinal tamponade is a useful adjunct to pars plana vitrectomy (PPV) in the treatment of complex vitreoretinal conditions. Siluron2000, a modified silicone oil product containing an additional small, high molecular weight and low viscosity, very-long-chain silicone molecule, was developed to reduce post-operative silicone oil emulsification, a non-infrequent complication that occurs with low molecular weight silicone oil. This study was designed to assess the safety and efficacy of long-term Siluron2000.

Patients and Methods: This was a single-center, retrospective, observational study. All consecutive patients undergoing PPV with intravitreal Siluron2000 injection between January 2017 and September 2019 with at least 6-month follow-up were identified based on billing codes.

Results: A total of 57 eyes of 57 consecutive patients comprising 51 cases of proliferative vitreoretinopathy and 6 cases of recurrent full thickness macular hole were included. Median follow-up was 18 months. Emulsification occurred in 9 patients (15\%). Retinal re-attachment was achieved in 47 patients (82\%). The median time without silicone oil emulsification was 17 months. Of the cases with residual retinal detachment (RD) despite intravitreal Siluron2000, 4 (7\%) were total RDs and $6(10 \%)$ were inferior RDs with attached macula. The most common complications were glaucoma 12 (21\%), cataract $11(19 \%)$, and epiretinal membrane $2(3.5 \%)$. There was no association between the duration of Siluron 2000 and visual outcomes.

Conclusion: Siluron2000 is an effective long-term tamponade agent in the treatment of complex vitreoretinopathy. Visual and anatomical outcomes are similar to those reported with higher mw silicone oil tamponade agents but with a lower emulsification rate.

Keywords: emulsification, proliferative vitreoretinopathy, silicone oil, tamponade, vitreoretinal surgery, long-term oil

\section{Introduction}

Silicone oil is commonly used as a vitreous substitute to provide retinal tamponade in the short- and long-term management of recurrent rhegmatogenous retinal detachments (RD) associated with giant retinal tears (GRT), viral retinitis, proliferative vitreoretinopathy (PVR), proliferative diabetic retinopathy (PDR) with tractional RD (TRD), chronic macular hole, and vitreoretinopathy secondary to trauma. It is also used as a primary retinal tamponade agent in patients who must
Correspondence: Mark S Mandelcorn

Tel + I 4166035885

Fax + I 4166035834

Email mark.mandelcorn@utoronto.ca 
return home by air travel shortly after surgery or are unable to comply with the positioning requirements of intraocular gas tamponade. ${ }^{1-3}$

A major barrier to long-term use of silicone oil is emulsification. Defined as the presence of small, noncoalescent oil bubbles in the vitreous cavity that also migrate into the anterior segment, emulsification may result in secondary open-angle glaucoma, hyperoleon formation, persistent inflammation, and band keratopathy that may continue to worsen even after removal of oil. Silicone oil emulsification may also mediate a pro-inflammatory milieu resulting in the recurrence of PVR, pre- and subretinal scarring, and possible retinal toxicity. ${ }^{1,2,4,5}$

Traditionally, silicone oils with a higher molecular weight $(\mathrm{mw})$ and viscosity were believed to result in less emulsification. Thus, the use of these oils, such as Silicone Oil 5000 centistoke (cSt), was favored for long-term or indefinite retinal tamponade. However, with the advent of minimally invasive vitrectomy surgery (MIVS), the use of higher viscosity oils has somewhat fallen out of favor due to the significantly higher injection and removal time required. $^{4-6}$ As well, there is a paucity of evidence to indicate a clinically significant difference in emulsification rates, for example, between $5000 \mathrm{cs}$ and $1000 \mathrm{cs}$ silicone oil. $^{4-7}$

To address these issues, a derivative of traditional silicone oil was manufactured in which a small amount of a very-long-chain silicone molecule was added to create what is commercially known as Siluron2000 (Labtician Ophthalmics, Oakville, Ontario, Canada). This product is purported to behave like a higher viscosity silicone product with less tendency to emulsify than other silicone oils with a similar viscosity due to its higher extensional viscosity. To-date, there are only a handful of publications reporting real-life data with the use of Siluron2000 for either short- or long-term internal retinal tamponade. ${ }^{7}$ The purpose of this study is to assess the long-term safety and efficacy of Siluron2000 in patients undergoing posterior segment surgical treatment of complex vitreoretinopathy.

\section{Materials and Methods}

Institutional Ethics Review Board approval was obtained for this retrospective observational review at Toronto Western Hospital, University Health Network, Toronto, Ontario. As per requirement from the Research Ethics Board at our institution, patient consent was not required due to the retrospective nature of the study. The study was compliant with confidentiality and compliance with the Declaration of Helsinki.

The charts of all consecutive patients who underwent 23-gauge pars plana vitrectomy (PPV) with intravitreal Siluron2000 between January 2017 and September 2019 were reviewed. Charts were identified based on the Ontario Health Insurance Plan (OHIP) billing code E148 (PPV), E142 (membrane stripping/peeling) and/or E151 (laser retinopexy). Patients were only included if Siluron2000 was used as part of PPV based on the operative record. Patients were excluded if they had less than 6 months of follow-up, or if the study eye had undergone prior PPV with the use of other types of oil. All surgical cases and follow-up visits were performed by a single vitreoretinal surgeon (MSM). Evidence of emulsification for all surgical patients was documented at each visit (ie post-operative day 1 , week 1 , week 4 , and then every 4-6 weeks for up to 6-12 months post-operatively as part of standard of care) by one examiner (primary surgeon) as the presence of oil bubbles in anterior chamber, clinically detectable bubbles on posterior capsule surface, or on the edges of the main bubble in the vitreous cavity.

\section{Surgical Technique}

PPV was performed using a standard 3-port 23-gauge system using a combination of macular contact lens viewing plus non-contact wide-angle viewing system (Resight 700, Carl Zeiss Meditec; Oberkochen, Germany). In all cases, patients received Siluron2000 (Labtician Ophthalmics, Oakville, Ontario, Canada) as a tamponade agent and 90$95 \%$ oil fill was achieved. When removing silicone oil, 3-6 fluid-air exchanges were performed until no residual floating oil bubbles were observed intra-operatively.

\section{Data Collection}

Compiled data including age, gender, initial diagnosis prior to Siluron2000 insertion, number of months with intact intravitreal Siluron2000, duration of follow-up, logarithm of minimum angle of resolution ( $\log M A R)$ of corrected distance visual acuity (VA) at baseline, and at the last follow-up were documented. The $\log$ MAR values for visual acuity of "hand motion" and "counting fingers" were assigned 2.2 and 1.8, respectively, based on previously published literature. ${ }^{8}$

\section{Outcome Measures}

The primary outcome measures were as follows: (1) emulsification rates as measured by the appearance of 
emulsified oil droplets in the anterior chamber or on the posterior lens capsule, or on the edges of the main bubble in the vitreous (2) change in visual acuity (VA) in logMAR at first and last follow-up, and (3) anatomical outcome measures such as the state of retinal attachment or macular hole closure at the final follow-up visit. Complications were recorded, namely, elevation of intraocular pressure (IOP), development of macular pucker, cataract, development of glaucoma as measured by progressive optic nerve thinning requiring drops, and presence of corneal haze or band keratopathy.

\section{Statistical Analysis}

Distribution of continuous variables was examined using histograms, box-whisker plots and Kolmogorov-Smirnov tests. Numerical variables were summarized using descriptive measures using counts and percentages, means and standard deviation for normally distributed continuous variables as well as median and interquartile range (IQR) for not normally distributed data. Baseline and follow-up visual acuity were not normally distributed and analyzed using the Wilcoxon Signed Ranks test. Linear regression analysis was used to determine the association between final visual acuity with baseline visual acuity and duration of Siluron 2000 in the vitreous cavity. The SPSS 27 (Chicago, IL) software was used for the data analysis. A $p$ value of less than 0.05 was considered for statistical significance.

\section{Results}

A total of 57 eyes of 57 patients met the study criteria. The baseline demographics of these 57 eyes are listed in Table 1. The average patient age was 63 (SD 13.1, range 26-89) years; $35 \%$ of patients were female. 43 eyes (75\%) had a prior history of ocular surgery: 13 eyes (23\%) had a scleral buckle (SB), 23 eyes (40\%) had pars plana vitrectomy (PPV), and 7 eyes (12\%) had combined SBPPV. Fourteen eyes (24.5\%) had no prior posterior segment surgery. Thirty-three eyes (58\%) had a diagnosis of rhegmatogenous $\mathrm{RD}$. Other preoperative preceding diagnoses were PVR with retinal detachment in 5 eyes $(8.7 \%)$, GRT in 6 eyes $(10 \%)$, full thickness macular hole (FTMH) in 6 eyes $(10 \%)$, and tractional retinal detachment (TRD) in $7(12 \%)$. Siluron2000 was used in 51 eyes $(89 \%)$ that had PVR, and 6 eyes that had a large or recurrent stage 4 FTMH.

Three cohorts of eyes were identified: (1) eyes in which Siluron2000 was removed prior to 6 months $(4 / 57$,
Table I Baseline Patient and Clinical Characterises Prior to Siluron2000 Insertion

\begin{tabular}{|l|c|c|}
\hline Patient and Clinical Characteristics & n/Mean & $\% / S D$ \\
\hline Mean age, years & 63.1 & 13.1 \\
Female & 20 & 35 \\
\hline Prior retinal surgery & 43 & 75 \\
SB & 13 & 23 \\
PPV & 23 & 40 \\
SB/PPV & 7 & 12 \\
No previous surgery & 14 & 24 \\
\hline Initial Diagnosis & & \\
RRD & 33 & 58 \\
PVR-RD & 5 & 9 \\
GT-RD & 6 & 10 \\
FTMH & 6 & 10 \\
TRD & 7 & 12 \\
\hline Pre-Siluron2000 Diagnosis & & \\
FTMH & 6 & 11 \\
PVR & 51 & 89 \\
\hline
\end{tabular}

Abbreviations: SB, scleral buckle; PPV, pars plana vitrectomy; RRD, rhegmatogenous retinal detachment; PVR-RD, proliferative vitreoretinopathy - retinal detachment; GT-RD, giant-tear with retinal detachment; FTMH, full-thickness macular hole; TRD, tractional retinal detachment; PVR, proliferative vitreoretinopathy.

7\%), (2) eyes in which Siluron2000 was removed after 6 months, but before the end of their follow-up period $(5 / 57$, $9 \%$ ), and (3) eyes with retained Siluron2000 for the duration of the follow-up period $(48 / 57,84 \%)$. The average follow-up time was 18 months (range 6 to 30 months). Emulsification occurred in 9 patients (16\%, Table 2), and the median time with non-emulsified intravitreal Siluron2000 was 17 months (IQR 12-21).

The median logMAR VA at baseline was 2.2 (IQR 1.72.5), which significantly improved to 1.6 (IQR 1.2-1.7) at last follow-up $(\mathrm{z}=-2.93, \mathrm{p}=0.003$, Table 2$)$. At the last follow-up, 53\% (30/57) of patients had ambulatory vision of at least counting fingers. Retinal re-attachment was achieved in 47 eyes (82\%). Among the cases with residual or recurrent RD despite intravitreal Siluron2000, 4 eyes (7\%) experienced total RD and 6 eyes $(10 \%)$ experienced inferior $\mathrm{RD}$ with attached macula. Five of the 6 eyes $(83.3 \%)$ with FTMH were able to achieve closure. The most common complications were glaucoma in 12 eyes (21\%) requiring long-term glaucoma drops, cataract in 11 eyes (19\%), transient elevation of intraocular pressure (IOP) in 4 eyes $(7 \%)$, and epiretinal membrane formation in 2 eyes (3.5\%).

There was no association between the duration of Siluron2000 in the vitreous cavity and visual acuity at 
Table 2 Summary of Primary Outcomes and Long-Term Complications Following Siluron2000 Insertion

\begin{tabular}{|c|c|c|}
\hline Variable & $\begin{array}{l}\mathrm{n} / \\
\text { Median }\end{array}$ & $\% / I Q R$ \\
\hline Follow-up duration, months & 18 & $15-22$ \\
\hline Duration with intact Siluron2000, months & 17 & $|2-2|$ \\
\hline \multicolumn{3}{|l|}{ Anatomical Outcomes } \\
\hline Retinal reattachment & 47 & 82 \\
\hline Recurrent detachment under oil & 10 & 17 \\
\hline Inferior RD & 6 & 10 \\
\hline Total RD & 4 & 7 \\
\hline \multicolumn{3}{|l|}{ Full thickness macular hole $(n=6)$} \\
\hline Macular hole closure & 5 & 83 \\
\hline Visual acuity at last follow-up, logMAR & 1.6 & $1.2-1.7$ \\
\hline \multicolumn{3}{|l|}{ Time to Siluron 2000 removal } \\
\hline$\leq 6$ months & 6 & 11 \\
\hline$>6$ months & 3 & 5 \\
\hline \multicolumn{3}{|l|}{ Complications } \\
\hline Silicone oil emulsification & 9 & 16 \\
\hline Corneal haze & I & 2 \\
\hline Epiretinal membrane & 2 & 4 \\
\hline Cystoid macular edema & 1 & 2 \\
\hline Cataract & 11 & 19 \\
\hline Elevated intraocular pressure $(\mathrm{IOP},>20)$ & 4 & 7 \\
\hline $\begin{array}{l}\text { Patient requiring glaucoma drops to } \\
\text { control IOP }\end{array}$ & 12 & 21 \\
\hline
\end{tabular}

Abbreviations: RD, retinal detachment; logMAR, logarithm of the minimum angle of resolution.

last follow-up after adjusting for baseline visual acuity using linear regression analysis (Table 3 ).

\section{Discussion}

In the surgical management of complex vitreoretinopathies, silicone oil tamponade is often desirable due to its long-term stability. Excellent functional and anatomic outcomes are reported with the use of silicone oil in RDs complicated by PVR, GRT, penetrating ocular trauma, and chronic FTMHs. ${ }^{9-13}$ However, gradual oil emulsification in the vitreous cavity with or without other complications, such as corneal decompensation, early cataract formation, and uncontrolled glaucoma, may prompt earlier oil removal surgery. ${ }^{14-18}$

Silicone oils with higher viscosities are thought to be more resistant to emulsification and are preferred for longterm tamponade. ${ }^{18}$ Viscosity is reported in units of millipascal second (mPas) or centistoke $(\mathrm{cSt})$, which are measurements of dynamic vs kinematic viscosity, respectively. For a given oil with a specific gravity close to 1 $\left(\mathrm{mPas} / \mathrm{cSt}\right.$ or $\left.\mathrm{g} / \mathrm{cm}^{3}\right), 1 \mathrm{mPas}$ is the equivalent of $1 \mathrm{cSt}$. Siluron2000 is a lower viscosity silicone oil (2000-2400 mPas, specific gravity $0.97 \mathrm{~g} / \mathrm{cm}^{3}$ ), consisting of $95 \%$ Siluron 1000 with additive high-molecular-weight components (5\% Polydimethylsiloxane, PDMS), which has been reported to have comparable anatomical and functional outcomes to Siluron5000 (5500 mPas) with better ease of surgical injection and removal. ${ }^{2,18}$ Currently, no published studies address the long-term tolerability, safety, and efficacy of Siluron2000. In this study, we attempted to address the long-term anatomical and functional outcomes and complications associated with Siluron2000.

Our findings suggest that $84 \%$ of eyes had intact Siluron 2000 over the duration of the follow-up period ( 6 to 31 months). Of the $16 \%$ of eyes, which required oil removal prior to the end of the follow-up period, $11 \%$ underwent oil removal prior to 6-months' follow-up post Siluron insertion, while $5 \%$ required removal after 6 months, but before the end of the follow-up period. This is a lower rate compared to reports using similar viscosity oils, namely, Densiron 68 (Fluoron Co, Neu-Ulm, Germany) (1400 mPas) with an emulsification rate of $42 \%$ in 33 eyes by 12 weeks, ${ }^{19}$ and OxaneHD (3300 $\mathrm{mPas}$ ) with a $52 \%$ emulsification rate over 26 months in $52 \%$ of 25 eyes with RDs. ${ }^{20-22}$ Variable rates of emulsification have also been reported for oils such as Silicone 1000 and $5000 \mathrm{cSt}$ ranging from $4 \%$ to $56 \%$ measured by the presence of oil droplets in the vitreous cavity or the

Table 3 Linear Regression Analysis of the Association of Visual Acuity at Last Follow-Up with Other Variables in Eyes with Siluron 2000 Insertion

\begin{tabular}{|l|l|l|l|}
\hline Variable* & Beta (Standardized Coefficients) & $\mathbf{9 5 \%} \mathbf{C l}$ & P value*** \\
\hline Visual acuity at baseline, logMAR & -0.004 & $-0.22-0.021$ & 0.977 \\
Duration of intact Siluron2000 in the vitreous cavity & 0.162 & $-0.105-0.42$ & 0.233 \\
\hline
\end{tabular}

Notes: Results are reported from general linear regression model. **All variables used in the linear regression model are continuous variables, including the visual acuity at last follow-up. A larger logMAR is suggestive of worse vision. *Statistically significant $(p<0.05)$.

Abbreviation: logMAR, logarithm of the minimum angle of resolution. 
anterior segment or complications secondary to emulsification such as corneal decompensation and poorly controlled IOP. ${ }^{3,21,22}$

Anatomical re-attachment was achieved in $82 \%$ of eyes in our series with siluron-in-situ, which is comparable to that achieved with higher viscosity silicone oils. ${ }^{23,24}$ Morphis et $\mathrm{al}^{23}$ have reported $74 \%$ anatomic success with Oxane5700 (5000 mPas oil) in 50 eyes followed for at least 12 months. Lam et $\mathrm{al}^{24}$ reported $83 \%$ anatomic success with OxaneHD (3300 mPas) in 147 eyes with grade C PVR. We report a $17 \%$ retinal re-detachment under oil. FTMH closure was achieved in $83 \%$, and only one patient experienced an inferior recurrent retinal detachment. Prior ocular surgery has been found to be a significant predictor of long-term anatomic success after silicone oil removal. Each subsequent recurrent detachment lowers the success rate of anatomical reattachment following silicone removal by $61 \% .^{24}$ In our study, $75 \%$ of patients had undergone prior unsuccessful $\mathrm{RD}$ repair surgery.

The final ambulatory visual outcomes were similar in this study to previously described literature with similar viscosity oils. There was a statistically significant improvement in vision from median baseline logMAR VA was 2.2 to 1.6 at last follow-up. Others have also reported severe-to-moderately low baseline visual acuity, as would be expected with complex and complicated cases of vitreoretinopathy requiring silicone oil tamponade. ${ }^{19,20,23-25}$ In other studies, using Oxane $5700^{23}$ or Densiron $68,{ }^{25} 40-61 \%$ of eyes had ambulatory vision or better at last follow-up. Furthermore, we found that final VA was not associated with the duration of Siluron2000 tamponade, which suggests that long-term oil may not necessarily prognosticate worse visual outcomes.

The most common complications in our study were glaucoma in $21 \%$ of eyes, cataract progression in $19 \%$ of eyes, and transiently elevated intraocular pressure $(>20$ $\mathrm{mmHg}$ ) in $7 \%$ of eyes. Cataract progression is the most common complication observed in other studies examining high and low viscosity silicone oils. Densiron $68^{19,26}$ and HWS 46-3000 (3000 msPas) oils ${ }^{27}$ were reported to cause cataract progression in all phakic eyes by 6 months. $(100 \%)$. This rate is about $38 \%$ with OxaneHD, ${ }^{28}$ and $90 \%$ with very low viscosity silicone oil. ${ }^{29}$ Similarly, Scott et $\mathrm{al}^{22}$ reported new cataract formation in $50 \%$ and $56 \%$, glaucoma in $11 \%$ and $7 \%$, and corneal decompensation in $6 \%$ ad $11 \%$ of patients with silicone oil 1000 and $5000 \%$, respectively, at 1-year follow-up.

Transient IOP changes are a common complication of silicone oil use. One study ${ }^{26}$ with Densiron 68 found an $8 \%$ elevation in IOP in 42 eyes with complicated RRD, although elevated IOP was defined as an IOP $>30 \mathrm{mmHg}$. We defined elevated IOP as $>20 \mathrm{mmHg}$. Another study of Densiron $68^{19}$ that defined elevated IOP as $>20 \mathrm{mmHg}$ found 30\% elevation in 33 eyes with complicated RDs. In another study, Oxane $\mathrm{HD}^{27}$ was reported to result in elevated IOP in $14 \%$ of 28 eyes with complicated RRD. Overall, the rate of transient or controlled IOP elevation in this study is comparable to previous reports. Despite lower IOP in comparison to other studies, ${ }^{19,26,28}$ we observed a greater incidence of glaucoma requiring IOPlowering drops, defined as progression of visual field loss and retinal nerve fibre thinning. Several factors can indeed contribute to IOP elevation and glaucoma following PPV with or without the use of silicone oil: PPV has long been known to be an independent risk factor for glaucoma. ${ }^{30}$ Chronic inflammation, and the use of short- or long-term steroids for the management of post-operative inflammation are amongst other factors that could contribute to elevated IOP. ${ }^{31}$ Studies by Sandner and colleagues ${ }^{32,33}$ on Densiron 68 found glaucoma in 10\% of eyes initially and in $17 \%$ of eyes with complicated RRDs after a $12-$ month follow-up. Wickham et $\mathrm{al}^{34}$ found glaucoma in $6 \%$ of 18 eyes that had an OxaneHD tamponade for 3 months over a 6-month follow-up period.

Other less common complications observed in our study were epiretinal membrane formation in $4 \%$ of eyes, corneal haze in $2 \%$, and cystoid macular edema (CME) in $2 \%$, which were comparable or lower than complications found in studies examining silicone oils. Epiretinal membrane formation was seen in Rizzo et $\mathrm{al}^{27}$ in HWS 463000 oil in $9 \%$ of 32 eyes with complicated RD. Majid et $\mathrm{al}^{35}$ observed increased membrane formation and cystoid macular edema with emulsification of Densiron 68. A study using F6H8 found corneal opacification in 1/ 5 cases $^{36}$ with inferior RDs. Another study ${ }^{37}$ using C10F18 found corneal opacification in $9 \%$ of 33 eyes with complicated RD. Our data suggest that apart from the long-term complication of glaucoma secondary to elevated IOP ( $>20 \mathrm{mmHg}$ ), Siluron 2000 might be safe both in the short and long terms.

There are several limitations in this study. First, silicone oil emulsification was identified by the appearance of oil particles in the anterior chamber, on the surface of the IOL, 
or as particles on the larger posterior vitreous bubble. Previous studies have used ultrasound to identify and quantify the degree of oil emulsification. ${ }^{4}$ Secondly, our relatively small sample size carries a limited statistical power in the detection of additional predictors, and the power of regression analysis for this data is therefore low. The retrospective nature of this study as well as the relatively small sample size conferred inter-sample variability in the characteristics of RDs. As with other retrospective RD studies, cases varied in duration, severity of PVR, number of detached quadrants, and factors, such as patient compliance with post-operative positioning that could not be controlled for. Future studies involving a control group are required in order to compare the results with cases in which conventional low molecular weight silicone oil is adopted.

\section{Conclusion}

Overall, Siluron2000 is a promising alternative to higher viscosity silicone oils in complex vitreoretinopathies requiring long-term internal silicone oil tamponade. Glaucoma requiring IOP lowering drops and cataract progression were the most common adverse events in the long-term use of intraocular Siluron2000. Nevertheless, long-term Siluron2000 achieves similar retinal reattachment and functional visual outcomes for higher viscosity oils.

\section{Disclosure}

The authors report no conflicts of interest in this work.

\section{References}

1. Caramoy A, Hagedorn N, Fauser S, Kugler W, Groß T, Kirchhof B. Development of emulsification-resistant silicone oils: can we go beyond $2000 \mathrm{mPas}$ silicone oil? Invest Ophthalmol Vis Sci. 2011;52 (8):5432-5436. doi:10.1167/iovs.11-7250

2. Williams RL, Day MJ, Garvey MJ, et al. Injectability of silicone oil-based tamponade agents. Br J Ophthalmol. 2011;95(2):273-276. doi:10.1136/bjo.2010.192344

3. Miller JB, Papakostas TD, Vavvas DG. Complications of emulsified silicone oil after retinal detachment repair. Semin Ophthalmol. 2014;29 (5-6):312-318. doi:10.3109/08820538.2014.962181

4. Caramoy A, Fauser S, Kirchhof B. Early emulsification of silicone oil (2000 cs) in minimally invasive transconjunctival vitreoretinal surgery". Klin Monatsbl Augenheilkd. 2010;228:477-479.

5. Mandelcorn E, Howarth D, Mandelcorn M. Silicone oil-induced bilateral granulomatous uveitis. Can J Ophthalmol. 2010;45(3):288-289. doi:10.3129/i09-235

6. Barca F, Caporossi T, Rizzo S. Silicone oil: different physical properties and clinical applications. Biomed Res Int. 2014;2014(502143):1-7. doi: $10.1155 / 2014 / 502143$

7. Stalmans P, Pinxten AM, Wong DS. Cohort safety and efficacy study of Siluron2000 emulsification-resistant silicone oil and F4H5 in the treatment of full-thickness macular hole. Retina. 2015;35 (12):2558-2566. doi:10.1097/IAE.0000000000000647
8. Schulze-Bonsel K, Feltgen N, Burau H, Hansen L, Bach M. Visual acuities "hand motion" and "counting fingers" can be quantified with the Freiburg visual acuity test. Invest Ophthalmol Vis Sci. 2006;47 (3):1236-1240. doi:10.1167/iovs.05-0981

9. Shen Y-D, Yang C-M. Extended silicone oil tamponade in primary vitrectomy for complex retinal detachment in proliferative diabetic retinopathy: a long-term follow-up study. Eur J Ophthalmol. 2007;17 (6):954-960. doi:10.1177/112067210701700614

10. Cibis PA, Becker B, Okun E, Canaan S. The use of liquid silicone in retinal detachment surgery. Arch Ophthalmol. 1962;68(5):590-599. doi:10.1001/archopht.1962.00960030594005

11. Zivojnović R, Mertens DA, Peperkamp E. Liquid silicone in amotio surgery (II). Report on 280 cases-further development of the technic. Klin Monbl Augenheilkd. 1982;181(6):444-452.

12. Altan T, Acar N, Kapran Z. Transconjunctival 25-gauge sutureless vitrectomy and silicone oil injection in diabetic tractional retinal detachment. Retina. 2008;28(9):1201-1206. doi:10.1097/ IAE.0b013e3181853d $3 \mathrm{c}$

13. Schurmans A, van Calster J, Stalmans P. Macular hole surgery with Inner limiting membrane peeling, endodrainage, and heavy silicone oil tamponade. Am J Ophthalmol. 2009;147(3):495-500. doi:10.1016/j.ajo.2008.09.003

14. Ichhpujani P, Jindal A, Jay Katz L. Silicone oil induced glaucoma: a review. Graefes Arch Clin Exp Ophthalmol. 2009;247 (12):1585-1593. doi:10.1007/s00417-009-1155-x

15. Matić S, Suić SP, Biuk D. Influence of silicone oil tamponade after vitrectomy on intraocular pressure. Coll Antropol. 2013;37:227-235.

16. Heidenkummer HP, Messmer EM, Kampik A. Recurrent vitreoretinal membranes in intravitreal silicone oil tamponade. Morphologic and immunohistochemical studies. Ophthalmologe. 1996;93:121-125.

17. Kociok N, Gavranic C, Kirchhof B, Joussen AM. Influence on membrane-mediated cell activation by vesicles of silicone oil or perfluorohexyloctane. Graefes Arch Clin Exp Ophthalmol. 2005;243 (4):345-358. doi:10.1007/s00417-004-1039-Z

18. Zhao X-J, Tang -N-N, Lian Y, Liu B-Q, Li Y-H, Lu L. Analysis of the rates of emulsification in intraocular silicone oil tamponades of differing viscosities. Int $J$ Ophthalmol. 2020;13(5):761. doi:10.18240/ijo.2020.05.10

19. Duan A, She H, Qi Y. Complications after heavy silicone oil tamponade in complicated retinal detachment. Retina. 2011;31(3):547-552. doi:10.1097/IAE.0b013e3181eef2fd

20. Prazeres J, Magalhães O, Lucatto LFA, et al. Heavy silicone oil as a long-term endotamponade agent for complicated retinal detachments. Biomed Res Int. 2014;2014. doi:10.1155/2014/136031

21. Zafar S, Shakir M, Mahmood SA, et al. Injectability of silicone oil-based tamponade agents. Br J Ophthalmol. 2011;52:5432-5436. doi:10.1097/00006982-200104000-00002

22. Scott IU, Flynn HW Jr, Murray TG, Smiddy WE, Davis JL, Feuer WJ. Outcomes of complex retinal detachment repair using 1000- vs 5000-centistoke silicone oil. Arch Ophthalmol. 2005;123 (4):473-478. doi:10.1001/archopht.123.4.473

23. Morphis G, Irigoyen C, Eleuteri A, Stappler T, Pearce I, Heimann H. Retrospective review of 50 eyes with long-term silicone oil tamponade for more than 12 months. Graefes Arch Clin Exp Ophthalmol. 2012;250(5):645-652. doi:10.1007/s00417-0111873-8

24. Lam RF, Cheung BTO, Yuen CYF, Wong D, Lam DSC, Lai WW. Retinal redetachment after silicone oil removal in proliferative vitreoretinopathy: a prognostic factor analysis. Am J Ophthalmol. 2008;145(3):527-533. doi:10.1016/j.ajo.2007.10.015

25. Herbrig E, Sandner D, Engelmann K. Anatomical and functional results of endotamponade with heavy silicone oil - Densiron ${ }^{\circledR} 68-$ in complicated retinal detachment. Ophthalmic Res. 2007;39 (4):198-206. doi:10.1159/000104681 
26. Wong D, Meurs JCV, Stappler T, et al. A pilot study on the use of a perfluorohexyloctane/silicone oil solution as a heavier than water internal tamponade agent. $\mathrm{Br} J$ Ophthalmol. 2005;89(6):662-665. doi:10.1136/bjo.2004.055178

27. Rizzo S, Genovesi-Ebert F, Vento A, Cresti F, di Bartolo E, Belting C. A new heavy silicone oil (HWS 46-3000) used as a prolonged internal tamponade agent in complicated vitreoretinal surgery: a pilot study. Retina. 2007;27(5):613-620. doi:10.1097/01. iae.0000251228.33870.64

28. Rizzo S, Genovesi-Ebert F, Belting C, Vento A, Cresti F. A pilot study on the use of silicone oil-RMN3 as heavier-than-water endotamponade agent. Graefes Arch Clin Exp Ophthalmol. 2005;243 (11):1153-1157. doi:10.1007/s00417-005-0015-6

29. Kirchhof B, Wong D, van Meurs J, Hilgers RD, Macek M. Use of perfluorohexyloctane as a long-term internal tamponade agent in complicated retinal detachment surgery. Am $J$ Ophthalmol. 2002;133(1):95-101. doi:10.1016/S0002-9394(01)01295-8

30. Kornmann HL, Gedde SJ. Glaucoma management after vitreoretinal surgeries. Curr Opin Ophthalmol. 2016;27(2):125. doi:10.1097/ ICU.0000000000000238

31. Roberti G, Oddone F, Agnifili L, et al. Steroid-induced glaucoma: epidemiology, pathophysiology, and clinical management. Surv Ophthalmol. 2020;65(4):458-472. doi:10.1016/J.SURVOPHT HAL.2020.01.002
32. Sandner D, Engelmann K. First experiences with high-density silicone oil (Densiron) as an intraocular tamponade in complex retinal detachment. Graefes Arch Clin Exp Ophthalmol. 2006;244 (5):609-619. doi:10.1007/s00417-005-0110-8

33. Sandner D, Herbrig E, Engelmann K. High-density silicone oil (Densiron) as a primary intraocular tamponade: 12-month follow up. Graefes Arch Clin Exp Ophthalmol. 2007;245(8):1097-1105. doi:10.1007/s00417-006-0496-y

34. Wickham L, Tranos P, Hiscott P, Charteris D. The use of silicone oil-RMN3 (Oxane HD) as heavier-than-water internal tamponade in complicated inferior retinal detachment surgery. Graefes Arch Clin Exp Ophthalmol. 2010;248(9):1225-1231. doi:10.1007/s00417-010-1358-1

35. Majid MA, Hussin HM, Biswas S, Haynes RJ, Mayer EJ, Dick AD. Emulsification of Densiron-68 used in inferior retinal detachment surgery. Eye. 2008;22(1):152-157.

36. Vote B, Wheen L, Cluroe A, Teoh H, McGeorge A. Further evidence for proinflammatory nature of perfluorohexyloctane in the eye. Clin Experiment Ophthalmol. 2003;31(5):408-414. doi:10.1046/j.14429071.2003.00687.x

37. Bottoni F, Sborgia M, Arpa P, et al. Perfluorocarbon liquids as postoperative short-term vitreous substitutes in complicated retinal detachment. Graefes Arch Clin Exp Ophthalmol. 1993;231 (11):619-628. doi:10.1007/BF00921955
Clinical Ophthalmology

\section{Publish your work in this journal}

Clinical Ophthalmology is an international, peer-reviewed journal covering all subspecialties within ophthalmology. Key topics include: Optometry; Visual science; Pharmacology and drug therapy in eye diseases; Basic Sciences; Primary and Secondary eye care; Patient Safety and Quality of Care Improvements. This journal is indexed on PubMed

Submit your manuscript here: https://www.dovepress.com/clinical-ophthalmology-journal

\section{Dovepress}

Central and CAS, and is the official journal of The Society of Clinical Ophthalmology (SCO). The manuscript management system is completely online and includes a very quick and fair peer-review system, which is all easy to use. Visit http://www.dovepress.com/ testimonials.php to read real quotes from published authors. 